Páginas: 91-107

Recibido: 2019-04-23

Revisado: 2020-12-11

Aceptado: 2021-01-11

Preprint: 2021-01-15

Publicación Final: 2021-01-31 www.revistascientificas.us.es/index.php/fuentes/index

DOI: https://doi.org/10.12795/revistafuentes.2021.v23.i1.8878

\title{
Integración de las actividades STEM en libros de texto
}

\author{
Integration of STEM activities in textbooks
}

\author{
iD Cristian Andres Ferrada Ferrada \\ Universidad de Granada (España) \\ (iD) Danilo Antonio Díaz-Levicoy \\ Universidad Católica del Maule(Chile) \\ iD Francisco Javier Carrillo Rosúa \\ Universidad de Granada (España)
}

\begin{abstract}
Resumen
La perspectiva educativa STEM está en auge y es coherente con el enfoque competencial que incorporan las legislaciones educativas. El objetivo de este trabajo es analizar en qué medida los libros de texto de Ciencias de Educación Primaria en España y Chile se alinean con esta perspectiva STEM. Siguiendo una metodología de análisis de contenido, en los 12 textos de amplia difusión analizados se identificaron unas 462 actividades, siendo 164 (menos del 50\%) las que trabajan alguna área STEM. Dichas actividades se clasificaron según el modelo de análisis de currículos integrados, que plantea seis maneras de abordar la integración de una actividad de este tipo, observando que el enfoque conectado, muestra una mayor relevancia en los libros de texto españoles (50\%) y chilenos (46\%). Posteriormente, se clasificaron según una adaptación del enfoque de perspectivas de Educación Ambiental. Dentro de los 5 enfoques existentes, el experiencial (60) y el práxico (50) son los que logran concentrar la mayor cantidad de actividades. Los hallazgos dan cuenta de la necesidad de incrementar la relación entre las diversas disciplinas para lograr un verdadero enfoque STEM integrado.
\end{abstract}

\begin{abstract}
The STEM educational perspective is rising and it s consistent with the competence approach incorporated in educational law. The aim of this work is to analyze to what extent the Primary Education Sciences textbooks in Spain and Chile are aligned with this STEM perspective. Following a content analysis methodology, in the 12 widely disseminated analyzed textbooks, 462 activities were identified. Among them, 164 (less than 50\%) are related with, at least, one STEM area. These activities were classified according to the integrated curriculum analysis model, which proposes six ways to approach the integration of an activity. The "linked approach" shows a greater relevance in Spanish (50\%) and Chilean textbooks (46\%). In adition, the activities were classified according to an adaptation of the environmental education perspective approach. Among 5 existing approaches, the "experiential" (60) and the "praxique" (50) are the most prevalent approaches. The findings reveal the need to increase the relationship among disciplines to achieve a truly integrated STEM approach.
\end{abstract}

Palabras clave / Keywords

Enfoques de integración, Enfoque de perspectivas, Educación STEM, Libros de texto, Actividades, Educación Primaria. Integration approaches, Perspective approach, STEM education, Textbooks, Activities, Primary education. 


\section{Introducción}

La educación STEM (Ciencia, Tecnología, Ingeniería y Matemática) se entiende como la conceptualización de todas las disciplinas que conforma su acrónimo en inglés, con el propósito de desarrollar un trabajo cohesionado, donde el aprendizaje es integrado, mediante diversas propuestas para enfrentar problemas del mundo real (Sanders, 2009).

La preocupación por la educación STEM, especialmente en el último tiempo, ha provocado el aumento de estudios sobre esta temática en diferentes niveles educativos, y también en su relación con el género (Rossi y Barajas, 2015). Sin embargo, en los primeros años de enseñanza obligatoria son más escasos (Ortiz-Revilla, Greca y Adúriz-Bravo, 2018). Pese a estar comprobado que fomentar el trabajo bajo experiencias científicas de orden práctico y manipulativo con estudiantes de Educación Primaria afecta positivamente sus apreciaciones y disposición en disciplinas STEM. Además, favorece la creatividad, imaginación y el trabajo en equipo (Bybee y Fuchs, 2006).

Este enfoque educativo permite la formación de aprendizajes en áreas de ciencia, ingeniería, tecnología y matemática, las que deben aumentar sus experiencias de enseñanza desde los primeros años de educación, abordando los conocimientos mediante variadas experiencias (National Research Council [NRC], 2012, 2014). Sheehan, Hightower, Lauricella y Wartella (2018) señalan que el trabajo de los estudiantes a temprana edad, con actividades STEM, aumenta el interés por estas disciplinas, desarrollando diversas habilidades que facilitarán las elecciones de futuras carreras en el área científica y matemática. Tsupros, Kohler y Hallinen (2009) manifiestan que la educación STEM debe concebirse desde el punto de vista interdisciplinar para la generación de aprendizajes, motivación e interés, facilitando la enseñanza en contextos próximos.

En esta misma línea, Quigley y Herro (2016) reconocen que uno de los principales objetivos del trabajo de la Educación STEM es entregar herramientas a los estudiantes, las que ayudarán a superar dificultades, mediante la innovación, el trabajo colaborativo y la creatividad. DeJarnette (2018) plantea que este tipo de actividades proporcionan a los estudiantes un entorno natural para la colaboración, cooperación y desarrollo de la comunicación, siendo capaces de generar oportunidades para la aplicación de estrategias , en un diseño de ingeniería simplista en un ambiente educativo.

Thibaut et al. (2018) afirman que existe un escaso número de estudiantes en áreas STEM, motivando a aumentar su interés. Por tanto, resulta fundamental promover el desarrollo de la ciencia, considerando que el desinterés dificulta el acceso a carreras STEM (Convert, 2005; Sanders, 2009). La alfabetización de los estudiantes con estas disciplinas contribuye a desarrollar habilidades, con las cuales se enfrentará con mayores posibilidad de éxito a una sociedad que avanza rápido tecnológicamente (Bybee, 2013), logrando desenvolverse en los nuevos escenarios.

Actualmente, los currículos escolares siguen fragmentado las distintas disciplinas relacionados con la matemática, ciencia y tecnología. Dicha separación dificulta la implementación efectiva de la Educación STEM, si bien el uso de metodologías activas, como el Aprendizaje Basado en Proyectos, de fuerte carácter interdisciplinar, podría facilitarlo (Domènech-Casal, 2018). Particularmente, en el caso de España, El Ministerio de Educación Cultura y Deporte (MECD, 2014) plantea un currículo en base a competencias educativas, de forma tal de integrar los contenidos trabajados en las áreas del conocimiento. Así, encontramos que STEM se presenta como una forma de interpretar este desafío, ya que para el desarrollo de una competencia de este tipo, se precisa de un trabajo relacionado entre las competencias de ciencia, matemática y tecnología de forma transversal. A su vez, el Ministerio de Educación de Chile (MINEDUC 2009, 2012) impulsa el trabajo en aéreas del conocimiento que desarrollen la interacción con aplicaciones tecnologías, despertando habilidades de investigación científicas. De igual manera, en las Bases Curriculares (MINEDUC, 2012) se establece la necesidad de generar instancias de interdisciplinariedad entre asignaturas, es decir, vemos como las asignaturas de tecnología, matemática y ciencia pueden ser trabajadas de manera intencionada, siendo esta una de las características esenciales de la Educación STEM.

Así pues, en este artículo, nos centraremos en el análisis del grado de integración STEM que presentan las actividades propuestas en los libros de texto chilenos y españoles de Educación Primaria. Y es que, aunque el movimiento STEM toma especial relevancia en la Educación Secundaria (Fuentes y González, 2017), la etapa de Educación Primaria no deja de ser crucial, ya que, por ejemplo la perspectiva STEM, que corresponde con una corriente internacional, también está siendo incorporando a la legislación educativa a la que los libros de texto tienen que dar respuesta. Así, en el caso español, donde desde la implementación de la Ley Orgánica de Educación (MEC, 2006), el currículo tiene una marcada orientación competencial, incluyendo las pruebas de evaluación externa (Díaz-Levicoy et al., 2017), nos encontramos actualmente con 
una competencia que se alinea perfectamente con la perspectiva STEM: competencia matemática y competencias básicas en ciencia y tecnología (MECD, 2015). Así, según MECD

En una sociedad donde el impacto de las matemáticas, las ciencias y las tecnologías es determinante, la consecución y sostenibilidad del bienestar social exige conductas y toma de decisiones personales estrechamente vinculadas a la capacidad crítica y visión razonada y razonable de las personas. A ello contribuyen la competencia matemática y competencias básicas en ciencia y tecnología.

$\operatorname{MECD}(2015$, p. 6993)

En Chile, las directrices curriculares de Educación Primaria (MINEDUC, 2012) señalan el marco general y los objetivos de aprendizaje estableciendo que la ciencia, tecnología y matemática han adquiriendo una relevante importancia para la formación de los estudiantes, resultando primordial establecer conexiones entre estas áreas, desde espacios reales, donde se pueda apreciar una aplicación significativa de lo aprendido e incentivar la interdisciplinariedad.

\section{Importancia de los libros de texto}

Entre los diversos materiales de trabajo presentes en el proceso educativo, destacan los libros de texto porque influye en los diferentes niveles de formación. Entre algunas de sus funciones se destacan: 1) el apoyo al profesor sobre la organización del proceso de instrucción, ya que presentan temas organizados, sugiriendo ejemplos, ejercicios, evaluaciones, etc.; 2) para los estudiantes es un apoyo al que pueden acceder en cualquier momento; 3) a las familias les permite seguir el proceso de enseñanza (Díaz-Levicoy et al., 2017). De modo similar, Torres y Moreno (2008) destacan que el libro de texto es una forma de comunicar conocimientos de una disciplina específica. En esta línea, Font y Godino (2006) mencionan que este recurso simboliza, en sí, una fuente concreta de saberes, en el cual se agrupan variadas experiencias, ejemplos prácticos de trabajo.

Son y Diletti (2017) destacan el potencial del libro de texto para el desarrollo de aprendizajes. Sin embargo, resaltan la importancia de la interacción entre los estudiantes y profesores, siendo necesario que estos últimos, conozcan los diversos beneficios que trae para la enseñanza el trabajo continuo. Por su parte, Danisova (2007) afirma que los libros de texto representan una orientación curricular para el trabajo del profesor, entregan estrategias metodológicas, estructuras para el desarrollo académico del estudiante y propuestas evaluativas de los contenidos. Así mismo, Fadzil y Saat (2014) enfatizan su utilidad de las actividades que se proporcionan en los libros de texto, a través de los cuales es posible interactuar e indagar en ejemplos prácticos y potenciar las habilidades científicas; lo que sin lugar a duda mantiene al libro de texto como el recurso didáctico mayormente utilizado (Perales, 2006).

A través del estudio de Cornejo (2006) es posible ver la evolución de los libros de texto de ciencia, reflejado en el cambio de la forma de trabajo y la búsqueda de un rol activo de los estudiantes. De igual forma, Pérez (2018) desarrolla una búsqueda histórica, donde determina las metodologías trabajadas en la enseñanza de las ciencias y la evolución a la actual educación STEM.

Diez y Rojo (2018) consideran estos enfoques en la planificación de una propuesta de intervención, la cual busca que los estudiantes trabajen pasos tales como: analizar fenómenos científicos, investigar sobre consecuencias y beneficios de las interacciones, manipular o experimentar diferentes compuestos, entre otros.

\section{3. Áreas STEM}

El aprendizaje de la educación STEM, desde un punto de vista interdisciplinar, exige que los estudiantes comprendan la importancia de su aprendizaje, su relación con la matemática, ciencia, tecnología y otras áreas académicas. El desarrollo de un aprendizaje focalizado en fenómenos científicos, entrega al estudiante una visión integral de los problemas en general, considerando elementos de:

Ciencia. El desarrollo de las ciencias busca potenciar diversas competencias del área desde los primeros años de educación, mediante la investigación y experimentación, en áreas de la naturaleza, biología, química, reacciones físicas y el universo astronómico, las cuales les permitan comprender, manipular y disfrutar de la disciplina. En este sentido, las actividades de ciencias deben buscar desarrollar un pensamiento crítico, por medio de la resolución de problemas (DeJarnette, 2018). 
Tecnología. La educación STEM busca que los estudiantes comprendan cómo la sociedad se nutre del mundo artificial y tecnológico, para dar soluciones a los desafíos actuales. La educación STEM busca la alfabetización tecnológica para comprender fenómenos y su posterior aplicación de los variados productos generados.

Ingeniería. Esta disciplina es vista como la oportunidad de presentar soluciones basadas en el ingenio, en un ambiente de resolución de problemas o desafíos académicos, el cual involucra el diseño, la creación de productos, el trabajo sistemático de procesos, considerando diversas concepciones de la naturaleza y las ciencias. La ingeniería se nutre de elementos de matemática y ciencias, así como herramientas de la tecnología (NRC, 2009). Fomentar la ingeniería, a través de un trabajo en educación STEM, dotará a los estudiantes de habilidades específicas y funcionales, mediante las cuales generarán diseños para resolver los desafíos.

Matemática. La educación STEM busca incorporar en las actividades diversos conceptos trabajados en ámbitos de números, álgebra, estadística, geometría o aritmética. Esta disciplina posee un papel prioritario en el trabajo e interpretación científica, validez de resultados y aplicaciones tecnológicas. Dentro de PISA se define competencia matemática como la capacidad para identificar, comprender e implicarse en las matemáticas y aplicarlo a contextos próximos a los estudiantes (OCDE, 2006).

De esta forma y como plantea English y King (2015), al analizar de forma conjunta, logramos identificar el enfoque STEM de manera integrada, ya que la Ciencia es vista como una forma de conocimiento transversal que busca comprender el mundo que nos rodea. La Tecnología como una forma de adaptación y un medio que necesariamente considera los impactos sociales. Ingeniería como una forma de diseñar soluciones para responder a problemas reales y las Matemáticas como una manera de expresar una comprensión/análisis del mundo y los problemas auténticos a través de los números. Como se ha mencionado anteriormente, la legislación avala un tratamiento conjunto integrado de estos saberes desde una perspectiva competencial (MECD, 2015).

\section{Modelo de análisis de currículos integrados, el enfoque de perspectivas en educación ambiental y objetivo de la investigación}

Pero, ¿cómo analizar la perspectiva STEM en libros de texto de Educación Primaria? En este sentido, aunque la literatura en análisis de libros de texto en el ámbito científico es extensa (e.g Aguilera y Perales, 2018; Laya y Martínez, 2019; Perales, 2006), al igual que lo es en todo lo relativo a propuestas STEM (Domènech- Casal, 2018; Martín-Páez et al., 2019b; Quigley y Herro, 2016; Sheehan et al., 2018; Thibaut et al., 2018), hay lagunas en el análisis de la perspectiva STEM en los libros de texto del ámbito científico. Una de las razones es la ausencia de modelos adaptados adecuados. En este trabajo, tras la revisión de la literatura realizada se aprecia la potencialidad del modelo de análisis de currículos integrados (Gresnigt et al., 2014) y del enfoque de perspectivas en educación ambiental (Sauvé, 2006).

Gresnigt et al. (2014) proponen un modelo de enfoques de análisis de currículos integrados, describiendo elementos que caracterizan el grado de integración de áreas STEM de una actividad. Estos corresponden a diferentes niveles (aislado o fragmentada; conectada, anidadas, multidisciplinar, interdisciplinar y transdisciplinar) y, que en este trabajo, los consideramos para categorizar los ejercicios propuestos en los libros de texto, destacando la interacción entre las áreas de estudio para lograr el objetivo final de la actividad. Por otra parte Sauvé (2006) plantea el enfoque de perspectiva en educación ambiental, que observa los diferentes matices que deben integrarse para el desarrollo de actividades en consonancia con la reutilización de materiales y la inserción que estos presentan en el aprendizaje (Sauve, 2000). En nuestro caso, adaptamos estos niveles (enfoque experiencial, crítico, práxico, interdisciplinario, participativo y colaborativo), para buscar comprender de qué forma las actividades STEM se adaptan en una perspectiva crítica y amigable con la sociedad.

Así, pues en este trabajo se pretende analizar en qué medida los libros de texto de ciencias de Educación Primaria en un contexto hispanoamericano son coherentes con una perspectiva STEM. Para ello, se pondrá el foco en las actividades, el elemento de los libros de texto que condiciona más condiciona el aprendizaje de los estudiantes desde una perspectiva competencial (Son y Diletti 2017). Por tanto, se plantean dos objetivos en este trabajo son:

1. Identificar actividades que promuevan el desarrollo de al menos 1 actividad STEM.

2. Interpretar y clasificar las actividades STEM según las propuestas de Gresnigt et al. (2014) y Sauvé (2006). 


\section{Método}

Esta investigación sigue una metodología cualitativa y descriptiva (Hernández, Fernández y Baptista, 2010); específicamente se empela el método de análisis de contenido (López- Noguero, 2002) desde una perspectiva comparada al centrarnos en los libros de texto de dos países hispanoamericanos referentes, como son Chile y España.

La muestra objeto de estudio está conformada por 12 libros de texto de Ciencias de la Naturaleza y Ciencias Naturales de $1^{\circ}$ a $6^{\circ}$ curso de Educación Primaria chilena y española. La casi totalidad de estos textos pertenecen a una misma editorial presente en ambos países (SM) y que en el caso chileno corresponden a la selección de libros elegidos por los establecimientos (MINEDUC, 2012) y por ende son de distribución gratuita. La selección de estos libros se justifica pues, en el caso chileno por su muy amplia difusión entre los los centros escolares (Nova et al., 2019), lo que también se cumple en el caso español porque SM es una de las tres editoriales con mayor cuota de mercado de España (CNMC, 2019). El único texto que no es de la editorial SM es el texto chileno de la editorial crecer pensando y que se ha seleccionado por estar en la selección del MINEDUC (2012). Las referencias de estos textos, vigentes para la docencia en el curso académico 2018-2019, se observan en la Tabla 1.

Tabla 1

Datos libros de texto chilenos (T1 a T6) y españoles (T7 a T12) analizados

\begin{tabular}{|c|c|c|c|}
\hline Código & Autores & Titulo & Editorial \\
\hline $\mathrm{T} 1$ & Equipo pedagógico SM (2018) & Texto del estudiante. Ciencias Naturales $1^{\circ}$ básico & SM \\
\hline $\mathrm{T} 2$ & Gutiérrez y Ortiz (2018) & Texto del estudiante. Ciencias Naturales $2^{\circ}$ básico & SM \\
\hline T3 & Calderón y Gutiérrez (2018) & Texto del estudiante. Ciencias Naturales $3^{\circ}$ básico & SM \\
\hline T4 & Molina, Morales y Ortiz (2018) & Texto del estudiante. Ciencias Naturales $4^{\circ}$ básico & SM \\
\hline T5 & Valdés y Rojas (2017) & Ciencias Naturales $5^{\circ}$ básico. Texto del estudiante & $\begin{array}{l}\text { Crecer } \\
\text { pensando }\end{array}$ \\
\hline T6 & Morales, Ortiz y Valdebenito (2017) & Ciencias Naturales $6^{\circ}$ básico. Texto del estudiante & SM \\
\hline T 7 & Cáliz, S. (2015) & $\begin{array}{l}\text { Texto del estudiante. Ciencias de la naturaleza } 1^{\circ} \\
\text { Primaria }\end{array}$ & SM \\
\hline T 8 & Garín, Hidalgo y Moratalla (2015) & $\begin{array}{l}\text { Texto del estudiante. Ciencias de la naturaleza } 2^{\circ} \\
\text { Primaria }\end{array}$ & SM \\
\hline T9 & Soria, Navarro y López (2014) & $\begin{array}{l}\text { Texto del estudiante. Ciencias de la naturaleza } 3^{\circ} \\
\text { Primaria }\end{array}$ & SM \\
\hline T 10 & Soria y Pueyo (2015) & $\begin{array}{l}\text { Texto del estudiante. Ciencias de la naturaleza } 4^{\circ} \\
\text { Primaria }\end{array}$ & SM \\
\hline T 11 & $\begin{array}{l}\text { Pueyo, Hidalgo, González, Peña, y } \\
\text { Navarro (2014) }\end{array}$ & $\begin{array}{l}\text { Texto del estudiante. Ciencias de la naturaleza } 5^{\circ} \\
\text { Primaria }\end{array}$ & SM \\
\hline T 12 & $\begin{array}{l}\text { Pueyo, González, Hidalgo y Moratalla } \\
\text { (2015) }\end{array}$ & $\begin{array}{l}\text { Texto del estudiante. Ciencias de la naturaleza } 6^{\circ} \\
\text { Primaria }\end{array}$ & SM \\
\hline
\end{tabular}

Para analizar las actividades se diseñaron dos instrumentos que incluían 7 (Tabla 2) y 6 (Tabla 3) categorías, respectivamente, extraídas del modelo de análisis de currículos integrados (Gresnigt et al., 2014) y del enfoque de perspectivas en educación ambiental (Sauvé, 2006). Se establece que las categorías, dentro de cada modelo considerado, son excluyentes entre sí. Es decir, cada actividad, finalmente, solo puede asignarse a una categoría dentro de cada modelo. 
Tabla 2

Instrumento para la clasificación de actividades según el modelo de enfoques de análisis de currículos integrados

\begin{tabular}{|c|c|c|c|c|c|c|c|c|}
\hline $\begin{array}{c}\text { Activida } \\
\text { d } /\end{array}$ & $\begin{array}{c}\text { Código } \\
\text { libro/ } \\
\text { página. }\end{array}$ & $\begin{array}{l}\text { Actividad } \\
\text { No STEM. }\end{array}$ & $\begin{array}{c}\text { Aislado o } \\
\text { fragmentad } \\
\text { a. }\end{array}$ & Conectada & Anidada & $\begin{array}{c}\text { Multi- } \\
\text { disciplinar }\end{array}$ & $\begin{array}{c}\text { Inter- } \\
\text { disciplinar }\end{array}$ & $\begin{array}{c}\text { Trans- } \\
\text { disciplinar }\end{array}$ \\
\hline & & $\begin{array}{l}\text { No exista } \\
\text { participación } \\
\text { explicita de } \\
\text { las áreas } \\
\text { científicas, } \\
\text { la } \\
\text { tecnología, } \\
\text { ingeniería y } \\
\text { la } \\
\text { matemática, } \\
\text { los } \\
\text { proyectos o } \\
\text { tareas no } \\
\text { utilizan las } \\
\text { similitudes, } \\
\text { contextos o } \\
\text { herramienta } \\
\text { s de estas } \\
\text { áreas para } \\
\text { conseguir el } \\
\text { objetivo } \\
\text { propuesto. }\end{array}$ & $\begin{array}{c}\text { Una } \\
\text { enseñanza } \\
\text { separada de } \\
\text { un tema, es } \\
\text { vista como } \\
\text { una forma } \\
\text { tradicional } \\
\text { de } \\
\text { educación, } \\
\text { donde no } \\
\text { existe } \\
\text { transversalid } \\
\text { ad. }\end{array}$ & $\begin{array}{c}\text { Genera una } \\
\text { conexión } \\
\text { clara entre } \\
\text { dos áreas } \\
\text { de estudio, } \\
\text { facilitando la } \\
\text { deducción } \\
\text { en la } \\
\text { comprensió } \\
\text { n. }\end{array}$ & $\begin{array}{c}\text { Un } \\
\text { contenido } \\
\text { de una } \\
\text { asignatura } \\
\text { es } \\
\text { desarrolla } \\
\text { do en otra, } \\
\text { enriquecie } \\
\text { ndo la } \\
\text { disciplina } \\
\text { en donde } \\
\text { se busca } \\
\text { generar el } \\
\text { aprendizaj } \\
\text { e. }\end{array}$ & $\begin{array}{c}\text { Es } \\
\text { necesario } \\
\text { que dos o } \\
\text { más } \\
\text { asignaturas } \\
\text { planifiquen } \\
\text { una unidad } \\
\text { de trabajo, } \\
\text { la cual es } \\
\text { tratada sin } \\
\text { perder la } \\
\text { identidad de } \\
\text { cada área } \\
\text { de estudio. }\end{array}$ & $\begin{array}{c}\text { No es } \\
\text { posible } \\
\text { distinguir } \\
\text { entre una } \\
\text { asignatura } \\
\text { específica, } \\
\text { trascendiend } \\
\text { o } \\
\text { habilidades } \\
\text { y } \\
\text { conocimient } \\
\text { os de } \\
\text { diversas } \\
\text { áreas de } \\
\text { estudio } \\
\text { relacionadas } \\
\text { entre sí. }\end{array}$ & $\begin{array}{c}\text { Las } \\
\text { actividades } \\
\text { son puestas } \\
\text { al servicio } \\
\text { de la } \\
\text { comprensió } \\
\text { n del mundo } \\
\text { real. Los } \\
\text { contenidos } \\
\text { curriculares } \\
\text { transciende } \\
\text { n las áreas } \\
\text { de estudio } \\
\text { focalizadas } \\
\text { tradicionalm } \\
\text { ente en } \\
\text { contenidos } \\
\text { de } \\
\text { aprendizaje. }\end{array}$ \\
\hline 1 & $\mathrm{~T} 1 / 5$ & $X$ & & & & & & \\
\hline 2 & $\mathrm{~T} 1 / 25$ & & $X$ & & & & & \\
\hline
\end{tabular}

Tabla 3

Instrumento para la clasificación de actividades según el Enfoque de Perspectiva en Educación Ambiental

\begin{tabular}{|c|c|c|c|c|c|c|c|}
\hline Actividad/ & $\begin{array}{c}\text { Código } \\
\text { libro/ } \\
\text { página. }\end{array}$ & $\begin{array}{l}\text { Actividad } \\
\text { No STEM. }\end{array}$ & $\begin{array}{c}\text { Enfoque } \\
\text { experiencial }\end{array}$ & $\begin{array}{c}\text { Enfoque } \\
\text { crítico }\end{array}$ & $\begin{array}{l}\text { Enfoque } \\
\text { práxico }\end{array}$ & $\begin{array}{c}\text { Enfoque } \\
\text { interdisciplinario }\end{array}$ & $\begin{array}{c}\text { Enfoque } \\
\text { participativo } \\
y \\
\text { colaborativo }\end{array}$ \\
\hline & & $\begin{array}{l}\text { No exista } \\
\text { participación } \\
\text { explicita de } \\
\text { las áreas } \\
\text { científicas, la } \\
\text { tecnología, } \\
\text { ingeniería y la } \\
\text { matemática, } \\
\text { los proyectos } \\
\text { o tareas no } \\
\text { utilizan las } \\
\text { similitudes, } \\
\text { contextos o } \\
\text { herramientas } \\
\text { de estas } \\
\text { áreas para } \\
\text { conseguir el } \\
\text { objetivo } \\
\text { propuesto. }\end{array}$ & $\begin{array}{lr}\text { Descubre los } \\
\text { aprendizajes a } \\
\text { través de } \\
\text { contextos reales } \\
\text { y próximos a los } \\
\text { estudiantes, } \\
\text { donde sean } \\
\text { capaces de } \\
\text { experimentar en } \\
\text { primera persona, } \\
\text { para su posterior } \\
\text { asimilación del } \\
\text { aprendizaje, } \\
\text { proporcionando } \\
\text { una oportunidad } \\
\text { de crear espacios } \\
\text { para construir } \\
\text { enseñanzas en } \\
\text { base a las } \\
\text { experiencias } \\
\text { previas. }\end{array}$ & $\begin{array}{l}\text { Identifica un } \\
\text { problema y da } \\
\text { una solución, } \\
\text { reconoce los } \\
\text { errores de } \\
\text { procesos que } \\
\text { presentan la } \\
\text { actividad, de } \\
\text { igual forma } \\
\text { utilizar la } \\
\text { limitación de } \\
\text { un problema, } \\
\text { en beneficio } \\
\text { del } \\
\text { aprendizaje. }\end{array}$ & $\begin{array}{l}\text { Relación } \\
\text { directa, a } \\
\text { través de la } \\
\text { reflexión en las } \\
\text { diferentes } \\
\text { actividades de } \\
\text { trabajo } \\
\text { presentadas, } \\
\text { genera } \\
\text { asociación lo } \\
\text { entre y } \\
\text { reflexionado y } \\
\text { las acciones } \\
\text { producidas a } \\
\text { través de lo } \\
\text { experimentado. }\end{array}$ & $\begin{array}{l}\text { Integración de } \\
\text { variadas } \\
\text { disciplinas busca } \\
\text { la respuesta a } \\
\text { problemas } \\
\text { formulado. A } \\
\text { través de la } \\
\text { conjugación de } \\
\text { áreas de estudio, } \\
\text { se establece una } \\
\text { mejor } \\
\text { combinación de } \\
\text { los } \\
\text { conocimientos, } \\
\text { facilitando una } \\
\text { perspectiva } \\
\text { amplia de los } \\
\text { elementos } \\
\text { abordados en la } \\
\text { temática. }\end{array}$ & $\begin{array}{l}\text { Estrategia de } \\
\text { resolución } \\
\text { del } \\
\text { problema, } \\
\text { desarrollo de } \\
\text { trabajos en } \\
\text { comunidad, } \\
\text { genera } \\
\text { diferentes } \\
\text { nexos entre } \\
\text { los } \\
\text { integrantes } \\
\text { del grupo de } \\
\text { acción, } \\
\text { mediante lo } \\
\text { cual, } \\
\text { enriquecerán } \\
\text { las se } \\
\text { discusiones y } \\
\text { experiencias. }\end{array}$ \\
\hline
\end{tabular}

\begin{tabular}{llll}
\hline T2 & 15 & $X$ & $X$ \\
\hline T8 & 52 & & previas. \\
\hline
\end{tabular}

El procedimiento consistió en que los autores identificaran todas las actividades en los libros que conforman la muestra $(n=462)$. A continuación se analizaron e interpretaron las actividades en función de las categorizarías establecidas en el instrumento de análisis (tablas 2 y 3). Adicionalmente, a cada actividad se le asignó una única categoría según el modelo de Gresnigt et al. (2014) y otra en función del de Sauvé (2006). Cabe señalar 
que solo 164 actividades se estimaron que promuevan al menos una de las áreas de ciencia, tecnología, ingeniería o matemáticas (298 actividades no STEM). Finalmente se realizó un análisis de frecuencias de la categorización realizada.

\section{Resultados}

A continuación, presentamos los resultados del análisis de las 164 actividades consideradas como STEM, de un total de 462. Primero, se describen e interpretan actividades representativas de cada una de las categorías consideradas (tablas 2 y 3) a la luz del modelo de análisis de currículos integrados (Gresnigt et al., 2014). y posteriormente se hace lo propio según el enfoque de perspectivas adaptado de Sauvé (2000).

\subsection{Análisis según modelo de currículos integrados}

\subsubsection{Actividad no STEM}

Un ejemplo de este enfoque se muestra en la Figura 1. La actividad busca incentivar el trabajo colaborativo entre pares, donde el objetivo es construir una frecuencia como resultado de apretar una pelota de goma y la relación con las contracciones generadas en el cuerpo. Su clasificación se debe a que no existe una aplicación de la tecnología o la ingeniería al proceso de trabajo, el diseño de una solución al problema no existe, de igual forma el trabajo tecnológico carece de aplicabilidad en lo propuesto.

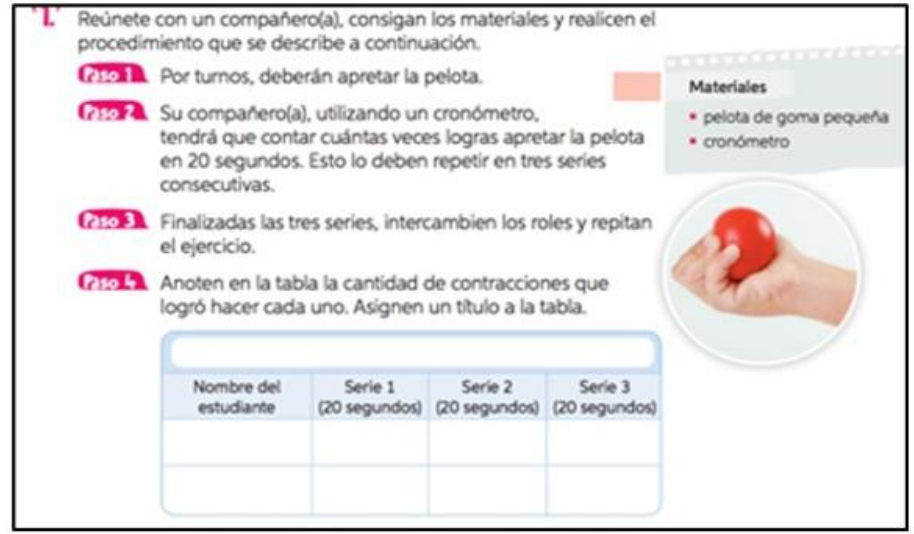

Figura 1. Actividad no STEM (T4, p. 62)

\subsubsection{Enfoque aislado}

La actividad mostrada en la (Figura 2, corresponde al enfoque aislada), ya porque el trabajo desarrollado no establece relación con otras asignaturas, su práctica es realizada de forma tradicional y no incluye el aporte de áreas de la tecnología, matemática o ingeniería para el logro del objetivo propuesto.

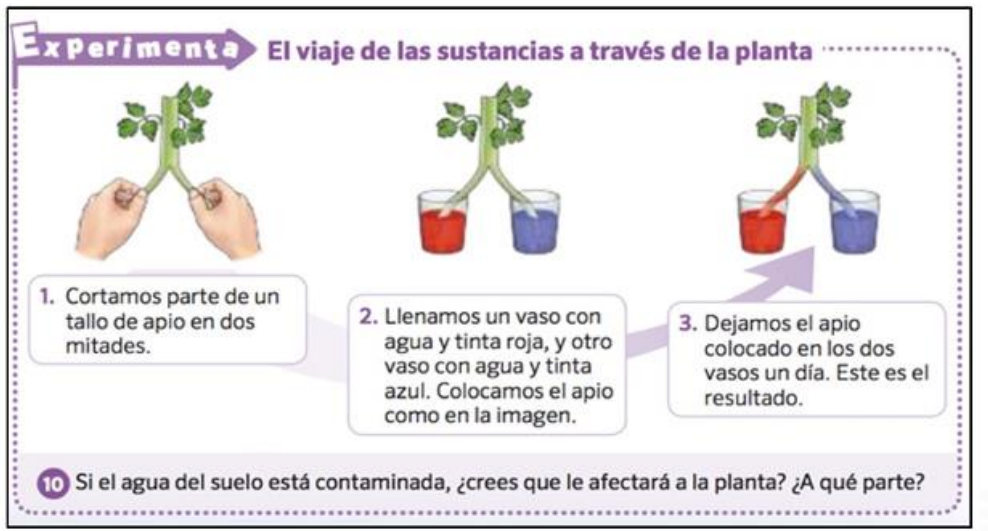

Figura 2. Actividad enfoque aislada (T8, p. 15) 


\subsubsection{Enfoque conectado}

La actividad que se observa en la Figura 3, busca que los estudiantes reflexionen, investiguen y tomen decisiones a lo largo de todo el proceso de trabajo. Se destaca la presencia de las 4 disciplinas, las cuales son abordadas de forma ordenada y sistemática, se identifica como mediante una planificación ingenieril, los estudiantes deben buscar lograr la mayor cantidad posible de energía para poder encender la bombilla. En esta actividad existe la conexión específica entre dos disciplinas, mediante el desarrollo de la experimentación en ciencias y la aplicación de recursos tecnológicos, es posible dar solución a la actividad.

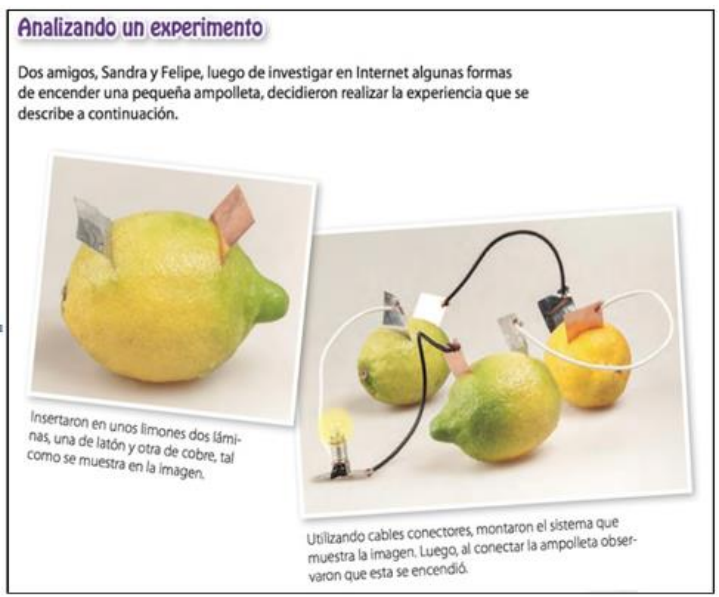

Figura 3. Actividad enfoque conectada (T5, p. 152)

\subsubsection{Enfoque anidado}

La siguiente actividad, presentada en la Figura 4, se considera clasificada dentro del enfoque de integración anidada, porque deben utilizar conocimientos de otras disciplinas para dar solución al problema que se formula. De manera guiada, seleccionan la información con la cual podrán organizar los pasos que llevarán a la concreción del proyecto científico. Mediante la matemática tendrán que registrar cada evolución de la planta, cantidad de agua necesaria y volumen de tierra para fortalecer la raíz de la planta y obtener diversas conclusiones, utilizarán la tecnología para complementar los conocimientos que harán que el proyecto funcione, buscando generar condiciones atmosféricas orientadas a beneficiar el éxito de la actividad. Cada una de estas actividades está siendo aplicada en la asignatura de ciencia mediante el desarrollo del método científico, dicha disciplina se nutre de las demás áreas para lograr el objetivo formulado.

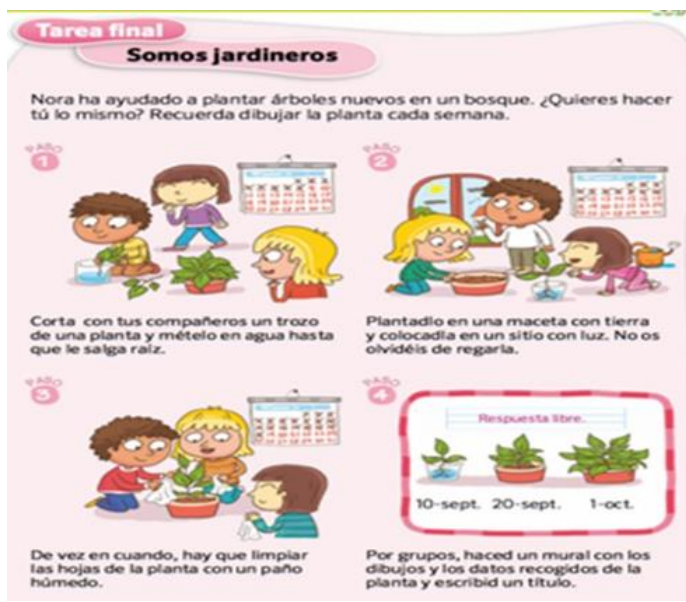

Figura 4. Actividad Enfoque anidada (T8, p. 21) 


\subsubsection{Enfoque multidisciplinar}

En la siguiente actividad (Figura 5) es posible observar como el desarrollo de un enfoque multidisciplinar es llevado a la práctica, aspectos de dos o más asignaturas son abordados, lo cual aumenta la motivación y el aprendizaje de los estudiantes, en base a una mejor compresión de los conceptos trabajados en la actividad. El objetivo de esta actividad es analizar, a través de la experimentación, la transformación de la energía. La ciencia se encuentra como eje articulador de la actividad y se inicia al momento de plantear una pregunta de investigación, formular y dar respuesta a la hipótesis que guiará el trabajo, desarrollar el experimento, registrar, interpretar y concluir los resultados para la posterior comunicación. La tecnología se manifiesta al utilizar los diversos diodos LED y motor eléctrico; la ingeniería se ejecutará en el diseño y montaje del generador; mientras la matemática se aplicará registrando el tiempo de uso del generador, duración e intensidad de la luz generada, cantidad y medidas de los materiales.

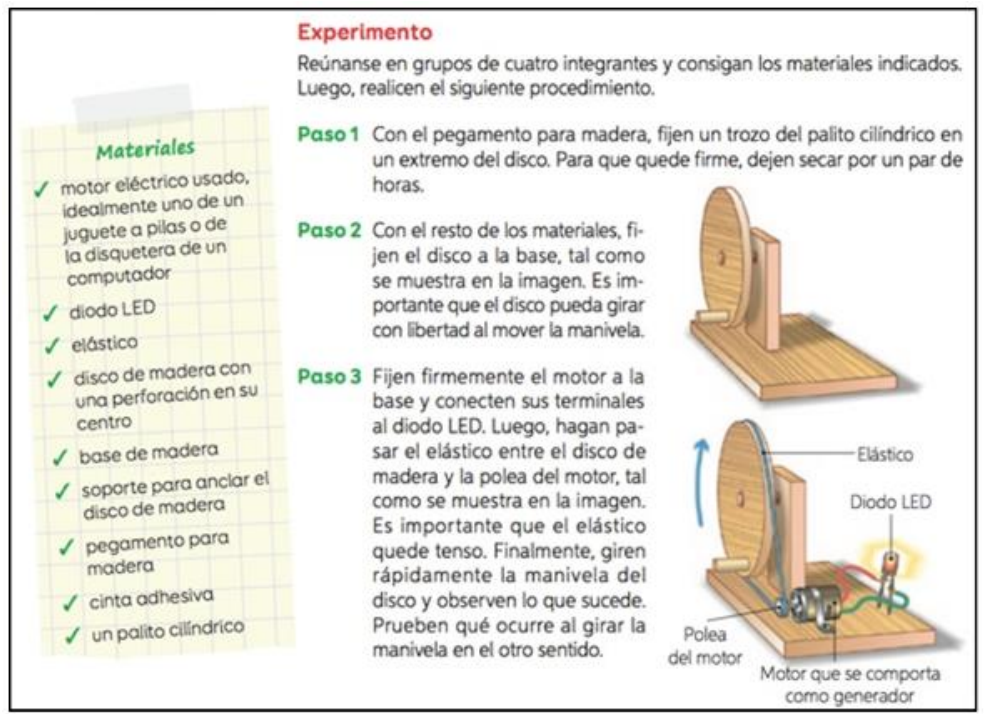

Figura 5. Actividad Enfoque multidisciplinar. (T6, p. 170)

En relación con los enfoques de integración interdisciplinar y transdisciplinar, no fue posible encontrar ese tipo de actividades en los 12 libros de texto analizados. Dentro de las posibles causas, se puede deber a que estos requieren un grado mayor de preparación en la concepción de la actividad.La cantidad y porcentaje de las actividades analizadas en los doce libros de texto se resume en la Tabla 4. Se observa que la mayor cantidad de actividades se encuentra en el libro de texto español de 6은 curso (30\% de actividades), seguida del $5 \div$ curso $(28,5 \%)$ y el libro que menor cantidad de actividades presenta el de $3^{\circ}$ curso. En el caso de los textos chilenos, presenten menor variación de la cantidad de actividades por curso, con mayor frecuencia en $6^{\circ}(23,7 \%)$ y $4^{\circ}$ $(21,8 \%)$ curso.

\section{Tabla 4}

Cantidad (y porcentaje) de las actividades analizadas en libros de texto chilenos y españoles de Educación Primaria

\begin{tabular}{llll}
\hline Curso & Libro de texto chilenos & Libro de texto españoles & Total \\
\hline $1^{0}$ & $36(13,7)$ & $16(8)$ & $52(11,3)$ \\
$2^{\circ}$ & $27(10,3)$ & $13(6,5)$ & $40(8,7)$ \\
$3^{0}$ & $39(14,9)$ & $6(3)$ & $45(9,7)$ \\
$4^{0}$ & $57(21,8)$ & $46(23)$ & $103(22,3)$ \\
$5^{0}$ & $41(15,6)$ & $57(28,5)$ & $98(21,2)$ \\
$6^{0}$ & $62(23,7)$ & $60(30)$ & $122(26,4)$ \\
Total & $262(100)$ & $200(100)$ & $462(100)$ \\
\hline
\end{tabular}

De igual forma, hemos clasificado entre actividades que buscan trabajar STEM y las que no (no STEM). Esto se resume en la Tabla 5, donde la mayor cantidad de actividades no STEM las encontramos coincidentemente en los cursos de mayor rango (6o curso), ya sea en los libros españoles (41 actividades) o chilenos (40 actividades). Del mismo modo, los cursos en donde se encuentra la mayor cantidad de actividades que es 
posible categorizarla como STEM es, en el caso chileno, en el curso $4^{\circ}$ con 27 actividades y en los libros españoles en $6^{\circ}$ con 19 propuestas de trabajo.

En relación con la clasificación, según los enfoques propuestos por Gresnigt et al. (2014), para analizar el nivel de integración de una actividad STEM. En los libros de texto chilenos (Tabla 6), vemos que la categoría de enfoque conectado es la más frecuente $(46,6 \%)$, evidenciando la necesidad de generar una relación entre asignaturas, para dar solución a lo formulado. En el caso contrario, es posible evidenciar que el enfoque multidisciplinar $(6,8 \%)$ es el menos trabajado, y solo visible a partir del $3^{\circ}$ curso; entendiendo la complejidad que supone lograr este tipo de clasificación para un ejercicio.

\section{Tabla 5}

Cantidad (y porcentaje) de las actividades clasificadas en STEM y no STEM de los libros de texto chilenos y españoles de Educación Primaria

\begin{tabular}{lllllll}
\hline \multirow{2}{*}{ Curso } & \multicolumn{2}{l}{ Actividades no STEM } & \multicolumn{3}{l}{ Actividades STEM } \\
\cline { 2 - 7 } & LT chilenos & LT españoles & Total & LT chilenos & LT españoles & Total \\
\hline $1^{0}$ & $22(13,8)$ & $13(9,4)$ & $35(11,7)$ & $14(13,6)$ & $3(4,9)$ & $17(10,4)$ \\
$2^{o}$ & $16(10,1)$ & $8(5,8)$ & $24(8,1)$ & $11(10,7)$ & $5(8,2)$ & $16(9,8)$ \\
$3^{\text {o }}$ & $25(15,7)$ & $2(1,4)$ & $27(9,1)$ & $14(13,6)$ & $4(6,6)$ & $18(11)$ \\
$4^{\circ}$ & $30(18,9)$ & $34(24,5)$ & $64(21,5)$ & $27(26,2)$ & $12(19,7)$ & $39(23,8)$ \\
$5^{0}$ & $26(16,4)$ & $39(28,1)$ & $65(21,8)$ & $15(14,6)$ & $18(29,5)$ & $33(20,1)$ \\
$6^{o}$ & $40(25,2)$ & $41(29,5)$ & $81(27,2)$ & $22(21,4)$ & $19(31,1)$ & $41(25)$ \\
Total & $159(100)$ & $139(100)$ & $298(100)$ & $103(100)$ & $61(100)$ & $164(100)$ \\
\hline
\end{tabular}

\section{Tabla 6}

Cantidad (y porcentaje) de las actividades clasificadas en STEM en libros de texto chilenos de Educación Primaria

\begin{tabular}{llllllll}
\hline Curso & $\mathbf{1}^{\mathbf{0}}$ & $\mathbf{2}^{\mathbf{o}}$ & $\mathbf{3}^{\mathbf{0}}$ & $\mathbf{4}^{\mathbf{0}}$ & $\mathbf{5}^{\mathbf{0}}$ & $\mathbf{6} \mathbf{0}$ & Total \\
\hline Aislado & $4(28,6)$ & $3(27,3)$ & $1(7,1)$ & $6(22,2)$ & $5(33,3)$ & $7(31,8)$ & $26(25,2)$ \\
Conectado & $9(64,3)$ & $4(36,4)$ & $4(28,6)$ & $14(51,9)$ & $6(40)$ & $11(50)$ & $48(46,6)$ \\
Anidado & $1(7,1)$ & $4(36,4)$ & $5(35,7)$ & $6(22,2)$ & $3(20)$ & $3(13,6)$ & $22(21,4)$ \\
Multidisciplinar & & & $4(28,6)$ & $1(3,7)$ & $1(6,7)$ & $1(4,5)$ & $7(6,8)$ \\
Total & $14(100)$ & $11(100)$ & $14(100)$ & $27(100)$ & $15(100)$ & $22(100)$ & $103(100)$ \\
\hline
\end{tabular}

En el caso de los textos españoles (Tabla 7), la mayoría de las actividades se relacionan con el enfoque conectado $(50,8 \%)$, es decir, promueve con mayor énfasis la interacción entre dos disciplinas para poder dar solución a un ejercicio. Es importante destacar que, para este análisis en los libros españoles no se encontraron actividades que pertenezcan a los últimos tres enfoques de mayor complejidad (multidisciplinar, interdisciplinar y transdisciplinar).

\section{Tabla 7}

Cantidad (y porcentaje) de las actividades clasificadas en STEM en libros de texto españoles de Educación Primaria

\begin{tabular}{llllllll}
\hline Curso & $\mathbf{1}^{\mathbf{0}}$ & $\mathbf{2} \mathbf{0}$ & $\mathbf{3} \mathbf{0}$ & $\mathbf{4}$ & $\mathbf{5}$ & $\mathbf{6}$ & Total \\
\hline Aislado & $1(33,3)$ & & & $3(25)$ & $6(33,3)$ & $9(47,4)$ & $19(31,1)$ \\
Conectado & $2(66,7)$ & $4(80)$ & $2(50)$ & $6(50)$ & $9(50)$ & $8(42,1)$ & $31(50,8)$ \\
Anidado & & $1(20)$ & $2(50)$ & $3(25)$ & $3(16,7)$ & $2(10,5)$ & $11(18)$ \\
Total & $3(100)$ & $5(100)$ & $4(100)$ & $12(100)$ & $18(100)$ & $19(100)$ & $61(100)$ \\
\hline
\end{tabular}

\subsection{Análisis según Enfoque de perspectiva}

\subsubsection{Enfoque experiencial}

En la actividad de la Figura 6 se observa que, a través del huerto colgante, mediante el reciclaje, los estudiantes aplican diferentes conocimientos STEM para resolver el problema. Se descubren aprendizajes en base a contextos y manipulación de objetivos reales, destacando la integración de conocimientos previos por los estudiantes, para lograr la efectividad del objetivo de la actividad. 


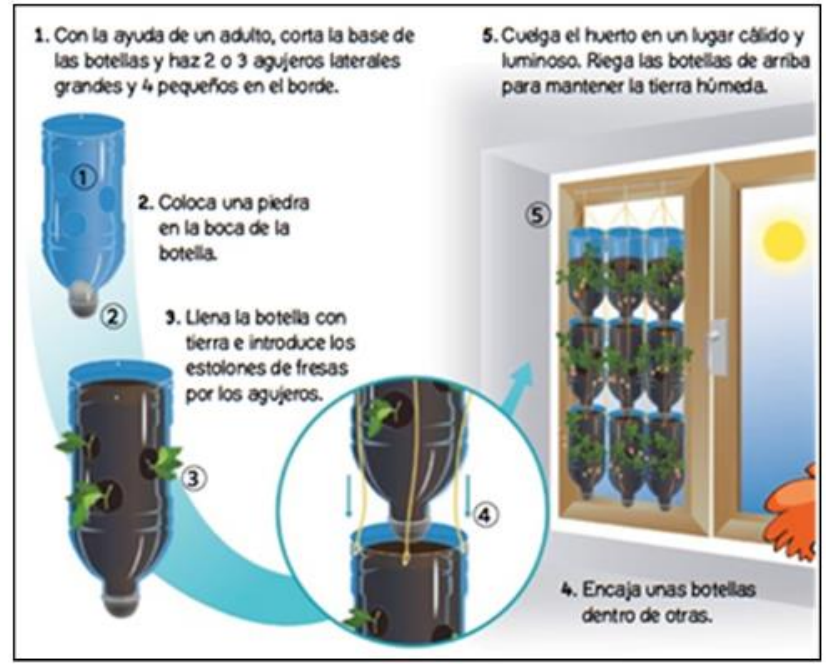

Figura 6. Enfoque experiencial (T5, p. 15)

\subsubsection{Enfoque crítico}

A través de la actividad de la Figura 7, y mediante la construcción de una cocina solar, es posible ver cómo y de forma crítica los estudiantes lograrán dar solución a un problema presente en diversas comunidades del mundo. En ella, energía es un bien escaso, junto con el desarrollo de la actividad y de forma paralela se genera una conciencia social sobre los recursos naturales; dicho aprendizaje beneficiará de forma directa la enseñanza de otras disciplinas académicas transversales a la ciencia y de orden formativos en valores sociales.

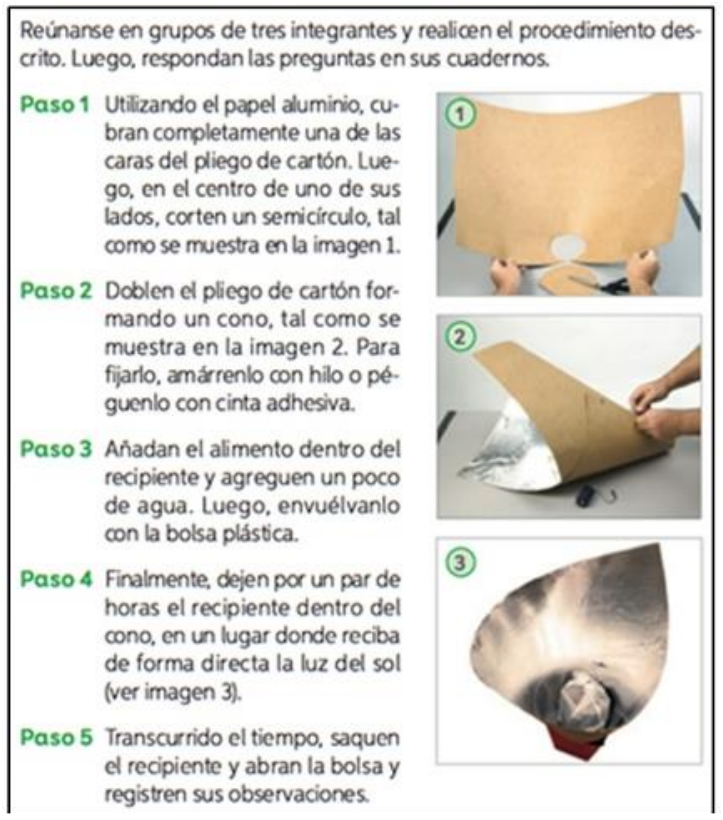

Figura 7. Enfoque crítico (T6, p. 177)

\subsubsection{Enfoque práxico}

En la actividad de la Figura 8 se busca, mediante la interacción práctica de los estudiantes y utilizando diferentes materiales reutilizados, representar esquemáticamente un aparato digestivo, reforzando 
aprendizajes futuros; a consecuencia del experimento realizado. Con ello, se espera comprender el funcionamiento y componentes del aparato digestivo a través de puesta en práctica de sus conocimientos.

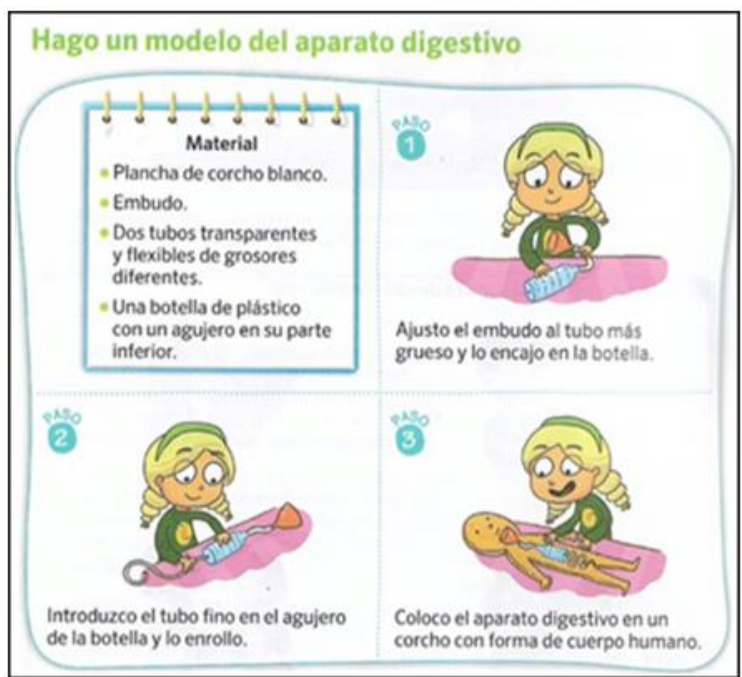

Figura 8. Enfoque práxico (T8, p. 52)

\subsubsection{Enfoque interdisciplinario}

En la Figura 9 se observa que, mediante la interacción de variados conocimientos disciplinarios, los estudiantes deben recrear diferentes ambientes, mediante implementos reciclados, reproducen el hábitat de las lombrices. Se evidencia el fortalecimiento de diversos conocimientos, matemáticos, tecnológicos y aplicación de soluciones mediante el ingenio, al ser una actividad que busca conjugar áreas transversales de la educación logra un mayor nivel fortalecimiento en la interacción de disciplinas académicas.

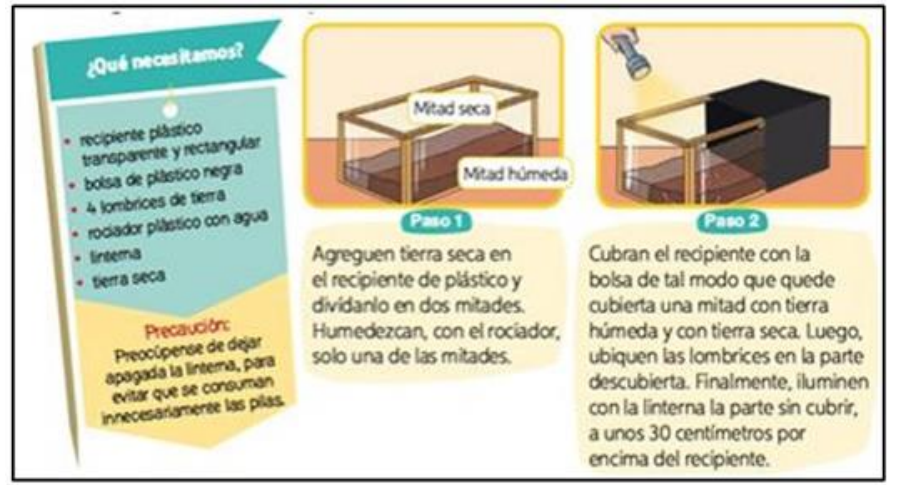

Figura 9. Enfoque interdisciplinario (T2, p. 118)

\subsubsection{Enfoque participativo y colaborativo}

A través de la actividad en la Figura 10, mediante el desarrollo de una actividad grupal, se busca que los estudiantes apliquen diversos conocimientos científicos y generen una interacción de saberes entre pares, de esta forma, dar solución al ejercicio; en la actividad se aprecia una interacción de elementos del medio ambiente, superficies de trabajo, factores de humedad y temperatura, logrando así el fortalecimiento de nuevos aprendizajes. 


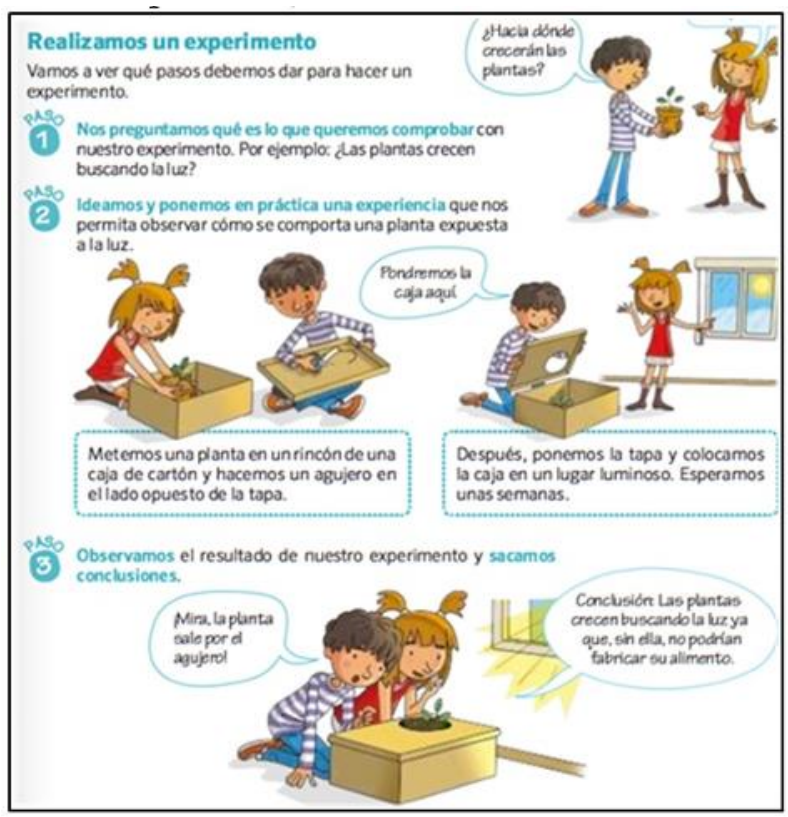

Figura 10. Enfoque participativo y colaborativo (T8, p. 18)

Para establecer un análisis sobre la cantidad de actividades y su categorización, según el enfoque de perspectivas presentes en las actividades STEM en textos españoles y chilenos, realizamos la Tabla 8. En ella, se manifiesta un claro predominio del enfoque experiencial (60 actividades: 37 en los textos chilenos y 23 en los españoles), teniendo que dicho enfoque busca utilizar las experiencias y conocimientos previos de los estudiantes, para de esta forma encontrar la solución al ejercicio inicial. De igual modo, el enfoque práxico es el segundo más trabajado, a consecuencia que los ejercicios analizados buscan en su gran mayoría la manipulación y la práctica de las actividades. En contraposición, encontramos que el enfoque interdisciplinar (12 actividades) es el menos observado, entendiendo que este tipo de categoría busca la conexión entre diversas áreas de la educación, lo cual representa un desafío en la construcción y posterior aplicación de la actividad.

\section{Tabla 8}

Resultados sobre cantidad de actividades según enfoque de perspectiva, adaptado de Sauvé, en libros de texto españoles y chilenos de Educación Primaria

\begin{tabular}{|c|c|c|c|c|c|c|c|c|c|c|c|c|c|c|c|}
\hline \multirow[b]{2}{*}{ Enfoques } & \multicolumn{7}{|c|}{ Texto chilenos } & \multicolumn{7}{|c|}{ Textos españoles } & \multirow[b]{2}{*}{ Total } \\
\hline & $1^{0}$ & $2^{0}$ & $3^{0}$ & $4^{\circ}$ & $5^{0}$ & $6^{\circ}$ & Total & $1^{0}$ & $2^{0}$ & $3^{0}$ & $4^{\circ}$ & $5^{\circ}$ & $6^{\circ}$ & Total & \\
\hline Experiencial & 5 & 3 & 6 & 13 & 3 & 7 & 37 & 1 & 1 & 2 & 5 & 8 & 6 & 23 & 60 \\
\hline Crítico & 0 & 0 & 1 & 0 & 4 & 3 & 8 & 0 & 1 & 0 & 1 & 0 & 3 & 5 & 13 \\
\hline Práxico & 2 & 4 & 2 & 6 & 6 & 9 & 29 & 2 & 2 & 1 & 4 & 6 & 6 & 21 & 50 \\
\hline Interdisciplinarios & 0 & 1 & 1 & 5 & 2 & 1 & 10 & 0 & 0 & 0 & 0 & 1 & 1 & 2 & 12 \\
\hline Participativo y colaborativo & 6 & 3 & 4 & 3 & 2 & 2 & 20 & 0 & 0 & 1 & 2 & 3 & 3 & 9 & 29 \\
\hline Total & 13 & 11 & 14 & 27 & 17 & 22 & 104 & 3 & 4 & 4 & 12 & 18 & 19 & 60 & 164 \\
\hline
\end{tabular}

\section{Discusión y conclusiones}

Este trabajo nos permite, en primer lugar, observar una dimensión del análisis de libros de texto ciertamente en crecimiento sobre las áreas STEM (Son y Diletti, 2017). En segundo lugar, identificamos las actividades que promueven el trabajo STEM (164) a través de los fundamentos teóricos que pretenden explicar la comprensión de esta incipiente área de estudio, así como la función que pueden desempeñar en los textos, dando respuesta a nuestro objetivo $n^{\circ} 1$. En tercer lugar, clasificamos las actividades, mediante el modelo de análisis de currículos integrados y enfoques de perspectivas.

La educación STEM, trabajada desde la integración de las diversas asignaturas presentes en el currículo escolar, es vista como una oportunidad de mejorar los procesos en la enseñanza y aprendizaje de los 
estudiantes. Por ello, es importante investigar las características de las actividades propuestas en los libros de texto, y el desarrollo de contenidos de orden específicos trabajados, transversalmente desde la ciencia, con lo cual se busca lograr una activación interdisciplinaria de otras disciplinas que ayudarán a fortalecer la dominio de los contenidos trabajados. En tal sentido, Merrill y Daugherty (2009) hacen referencia sobre el abordaje de las temáticas de una forma dinámica, y los contenidos específicos de una manera integradora mediante la actividad propuesta.

Una de las premisas consideradas para evidenciar la relevancia de las conexiones entre las asignaturas es trabajar bajo un enfoque integrador (Breiner, Harkness, Johnson y Koehler, 2012), el cual pueda mostrar un trabajo sistemático entre las áreas de aprendizaje. A su vez, los maestros, apoyados de los libros de texto pueden generar situaciones que apoyen la enseñanza y el aprendizaje. Por esto, el desafío está en buscar y entregar no solo conocimientos, sino tratar de trabajar sobre las prácticas del profesor y los desafíos pedagógicos a los cuales nos enfrentamos como sociedad (Fleer y Pramling, 2015).

Este trabajo proporciona un análisis de cómo se puede ver la educación STEM y las diferentes categorías que fueron analizadas sus actividades, centrándose más que solo la secuenciación de STEM en las propuestas evidenciadas en los textos (Lazonder y Harmsen, 2016).

Además, creemos que el modelo de análisis de currículos integrados (Gresnigt et al., 2014), al ser una propuesta que considera las formas de integrar las asignaturas STEM, logra mayores posibilidades que los estudiantes adquieran competencias necesarias en los escenarios actuales, reforzando la idea de concebir la educación desde el punto de vista interdisciplinar, las cuales se llevan a la práctica por medio del libro de texto (Tsupros, Kohler y Hallinen, 2009). De igual forma, se destaca la importancia de analizar constantemente la metodología de trabajo en el aula, favoreciendo el desarrollo de una indagación guiada, con el acompañamiento de los maestros, y fortaleciendo las habilidades científicas desde una temprana edad (Lazonder y Harmsen, 2016).

Respecto a los resultados del estudio, se observa que el enfoque conectado, muestra una mayor relevancia en los libros de texto españoles $(50,8 \%)$ y chilenos (46,6\%). Ante el escaso nivel de integración de las actividades observado, teniendo en cuenta que este enfoque representa solo el segundo nivel de un total de seis, los resultados son preocupantes. Sin embargo, el hecho de tener actividades que requieran la conexión de dos áreas de estudio evidencia un escenario positivo, ya que los estudiantes están expuestos a buscar estrategias para lograr la solución a los problemas.

De notoria importancia, es ver como de los 462 ejercicios propuestos en los 12 textos analizados, más del $50 \%$ no buscan potenciar el desarrollo de conocimientos STEM. Situación similar ocurre al analizar las actividades que promueven el aprendizaje de competencias STEM, las que muestran un progresivo aumento a medida que se avanzan en curso. De esta forma, vemos como la enseñanza de las ciencias en la primaria, trabaja desde los primeros años de escolaridad aspectos de la educación STEM. Sin embargo, al tener el área científica un potencial transdiciplinar, creemos la necesidad de aumentar de este tipo de actividades, apuntando así, el mejoramiento de conocimientos, habilidades y rendimiento en evaluaciones estandarizadas, tal como mencionamos la realidad de matemática y ciencia en el contexto de PISA.

Dentro del análisis, que busca conocer el nivel de integración, según los enfoques de perspectivas Sauvé (2006), en las 164 actividades clasificadas como STEM, se evidencia una baja presencia de los niveles que necesitan mayor relación con otras áreas de aprendizaje. Dentro de los 5 enfoques antes mencionados, el experiencial (60) y práxico (50) son los que logran concentrar la mayor cantidad de actividades. Una explicación para esta situación, se establece al ver que los ejercicios propuestos buscan incorporar los aprendizajes previos de los estudiantes, de esta forma los nuevos temas se amalgaman a los ya existentes, potenciando la observación de fenómenos y resultados de las actividades de manipulación científica. Con esta clasificación respondemos a nuestro objetivo no2, logrando clasificar las actividades seleccionados según los dos modelos propuestos.

De esta forma encontramos que existen diferentes grados de integración cuando se trata de un enfoque STEM (English y King, 2015). Además, parece que el grado de integración y el enfoque de perspectiva en educación ambiental puede estar relacionado con las propuestas existentes en los diferentes textos analizados, estableciendo conexiones explícitas y significativas entre las disciplinas STEM estudiadas.

$\mathrm{Si}$ bien este estudio no intentó evaluar la calidad, nuestros hallazgos brindan ejemplos de actividades STEM integrado unidad de instrucción con una perspectiva ambiental. Se requieren más estudios para comprender cómo el grado de integración se relaciona con la calidad de la instrucción y también con los resultados del aprendizaje de los estudiantes, sugiriendo la necesidad de involucran en mayores cantidades una instrucción integrada proporcionan un contexto de aprendizaje auténtico que establece conexiones claras entre disciplinas, reflejando los componentes clave de la educación STEM.

Nuestros hallazgos arrojan luz sobre cómo los textos de gran divulgación entre los estudiantes, carecen de una conexión con el fomento de educación STEM, Si bien los temas presentados anteriormente muestran una 
clara clasificación inicial al momento de categorizar las actividades, representan desafíos que enfrentan los maestros al momento de impartir y utilizar los textos como una forma de representación del currículo escolar, también dilucidar la manera que se trabaja la integración exitosa del trabajo en áreas STEM. Nuestro estudio evidencia la necesidad de incorporar y equilibrar las disciplinas STEM en el trabajo directo en el aula (Merril y Daugherty, 2009).

Finalmente, creemos que la integración de contenidos curriculares, específicamente en las actividades de ciencia trabajadas con estudiantes a través de los libros de texto, deben ser vistas como un motor decambio en la educación, ya que la necesidad de conjugar otras asignaturas y lograr el objetivo formulado para un ejercicio es la verdadera integración que se requiere en las aulas de clases. De igual forma, el continuo trabajo del maestro en actualizarse para ayudar en la indagación guiada es visto como prioritario para el éxito de cada propuesta de trabajo.

Hasta ese momento, sin embargo, este estudio actual puede actuar como un recurso para ayudar en las diversas prácticas educativas a comprender los desafíos que los y las docentes pueden enfrentar al tratar de llevar un enfoque STEM a sus aulas

\section{Apoyos}

\section{Beca CONICYT PFCHA 72190253}

\section{Referencias}

Breiner, J. M., Harkness, S. S., Johnson, C. C., y Koehler, C. M. (2012). What Is STEM? A Discussion About Conceptions of STEM in Education and Partnerships. School Science and Mathematics, 112(1), 3-11. Retrieved from https://dx.doi.org/10.1111/j.1949-8594.2011.00109.x 10.1111/j.1949-8594.2011.00109.x

Bybee, R. W. (2013). The case for STEM education: challenges and opportunities. Arlington, VA: National Science Teachers Association.

Bybee, R. W., y Fuchs, B. (2006). Preparing the 21st century workforce: A new reform in science and technology education. Journal of Research in Science Teaching, 43(4), 349-352. Retrieved from https://dx.doi.org/10.1002/ tea.20147 10.1002/tea.20147

Calderón, P., y Gutiérrez, S. (2018). Texto del estudiante. Ciencias Naturales 3ํBásico. Santiago: SM.

Cáliz, S. (2015). Texto del estudiante. Ciencias de la Naturaleza 10 Primaria. Murcia: SM.

Cornejo, J. (2006). La enseñanza de la ciencia y la tecnología en la escuela argentina (1880-2000): un análisis desde los textos. Enseñanza de las Ciencias, 24(3), 357-370.

Danisova, E. (2007). Política para la publicación de libros de texto en República de Eslovaquia. In MINEDUC (Ed.), Primer Seminario Internacional de Textos Escolares (p. 64-69). MINEDUC.

DeJarnette, N. K. (2018). Implementing STEAM in the Early Childhood Classroom. European Journal of STEM Education, 3(3). Retrieved from https://dx.doi.org/10.20897/ejsteme/3878 10.20897/ejsteme/3878

Díaz-Levicoy, D., Giacomone, B., y Arteaga, P. (2017). Caracterización de los gráficos estadísticos en libros de texto argentinos del segundo ciclo de Educación Primaria. Profesorado. Revista de Currículum y Formación del Profesorado, 21(3), 299-326.

Diez, M., y Rojo, D. (2018). Comprendiendo el origen y las consecuencias de la lluvia ácida desde una actividad STEM. Proyectos STEAM para la Educación Primaria. Fundamentos y aplicaciones prácticas, 259-278.

Domènech-Casal, J. (2018). Aprendizaje Basado en Proyectos en el marco STEM. Componentes didácticas para la Competencia Científica. Ápice. Revista de Educación Científica, 2(2), 29-42. Retrieved from https:// dx.doi.org/10.17979/arec.2018.2.2.4524 10.17979/arec.2018.2.2.4524

English, L. D., y King, D. T. (2015). STEM learning through engineering design: fourth-grade students' investigations in aerospace. International Journal of STEM Education, 2(1). Retrieved from https://dx.doi.org/ 10.1186/s40594-015-0027-7 10.1186/s40594-015-0027-7

Equipo Pedagógico SM. (2018). Texto del estudiante. Ciencias Naturales 1ํㅡásico. Santiago: SM.

Fadzil, H. M., y Saat, R. M. (2014). Enhancing STEM Education during School Transition: Bridging the Gap in Science Manipulative Skills. EURASIA Journal of Mathematics, Science and Technology Education, 10(3), 209-218. Retrieved from https://dx.doi.org/10.12973/eurasia.2014.1071a 10.12973/eurasia.2014.1071a Fernández-Martín, F.-D., Arco-Tirado, J.-L., Carrillo-Rosúa, F.-J., Hervás-Torres, M., Ruiz-Hidalgo, J.-F., y Romero-López, C. (2020). Making STEM Education Objectives Sustainable through a Tutoring Program. Sustainability, 12(16), 6653-6653. Retrieved from https://dx.doi.org/10.3390/su12166653 10.3390/su12166653 Fleer, M., y Pramling, N. (2015). A cultural-historical study of children learning science, cultural studies of science education. Dordrecht: Springer.

Font, V., y Godino, J. D. (2006). La noción de configuración epistémica como herramienta de análisis de textos matemáticos: su uso en la formación de profesores. Educação Matemática Pesquisa, 8, 67-98. Retrieved from 
https://bit.ly/2Luiivi

Fuentes, M., González, J., Garín, M., Hidalgo, J. M., Moratalla, V. . R., Taconis, R., Keulen, H. V., Gravemeijer, K., y Baartman, L. (2014). Necesidades formativas del profesorado de Secundaria para la implementación de experiencias gamificadas en STEM. Revista de Educación a Distancia, 54, 47-84. http:// dx.doi.org/10.6018/red/54/8

Gutiérrez, S., y Ortiz, P. (2018). Texto del estudiante. Ciencias Naturales 20 Básico. Santiago: SM. Hernández, R., Fernández, C., y Baptista, P. (2010). Metodología de la investigación. México: McGraw-Hill. Laya, P., y Martínez, C. (2019). La competencia científica en los libros de texto de educación primaria. Ápice. Revista de Educación Científica, 3(1), 71-83.

Lazonder, A. W., y Harmsen, R. (n.d.). Metanálisis del aprendizaje basado en la indagación: efectos de la orientación. Revisión de Investigación Educativa, 86(3), 681-718.

Martín-Páez, T., Aguilera, D., Perales-Palacios, F. J., y Vílchez-González, J. M. (2019). What are we talking about when we talk about STEM education? A review of literature. Science Education, 103(4), 799-822. Retrieved from https://dx.doi.org/10.1002/sce.21522 10.1002/sce.21522

Martín-Páez, T., Carrillo-Rosúa, J., Lupiañez-Gómez, J. L., y Vílchez-González, J. M. (2016). Análisis de las pruebas externas de evaluación de la competencia científico-tecnológica de 60 de Educación Primaria en España. Enseñanza de las Ciencias. Revista de investigación y experiencias didácticas, 37, 127-149.

MEC. (2006). Ley Orgánica de Educación (LOE) de 3 de mayo de 2006. In BOE (Vol. 106, p. 17158-17207). MECD. (2014). Real Decreto 126/2014, de 28 de febrero, por el que se establece el currículo básico de la Educación Primaria. Boletín Oficial del Estado, 52(1 de marzo de 2014), 19349-19420.

Ministerio de Educación. (2014). Real Decreto 126/2014, de 28 de febrero, por el que se establece el currículo básico de la Educación Primaria. In BOE (Vol. 52, p. 19349-19420).

Merrill, C., y Daugherty, J. (2009). The future of TE masters degrees: STEM. Trabajo presentado en la 70th Annual International Technology Education Association Conference. Louisville, Estados Unidos.

MINEDUC. (2009). Ley 20370. Ley General de Educación. Valparaíso: Cámara de Diputados. MINEDUC. (2012). Bases curriculares: Primero a Sexto básico. Santiago.

Molina, C., Morales, K., y Ortiz, P. (2018). Texto del estudiante. Ciencias Naturales 4o Básico. Santiago: SM. Morales, K., Ortiz, P., y Valdevenito, S. (2017). Texto del estudiante. Ciencias Naturales 6o Básico. Santiago: SM.

Nova, A., Huanquimilla, C., Vázquez, L., y Rebolledo, P. (2019). Política de textos escolares en Chile: Criterios de elegibilidad y consideraciones didácticas. Revista ciencias humanas y sociales, 35, 41-82.

NRC. (2009). Learning science in informal environments: people, places, and pursuits. In and others (Ed.), . Washington, DC: NAP.

OCDE. (2016). PISA 2015. Resultados clave. Paris.

Ortiz-Revilla, J., Greca, I. M., y Adúriz-Bravo, A. (2018). La Educación STEAM y el desarrollo competencial en la Educación Primaria. Proyectos STEAM para la Educación Primaria. Fundamentos y aplicaciones prácticas, 41-54.

Pérez, J. (2006). Un viaje en el tiempo por la alfabetización científica en España. Didácticas Específicas. Enseñanza de las Ciencias, 18(1), 13-30.

Pueyo, I., González, M., Hidalgo, J. M., y Moratalla, V. (2015). Texto del estudiante. Ciencias de la Naturaleza 6 - Primaria (and others, Ed.). Murcia: SM.

Pueyo, I., Hidalgo, A. J., González, M., Peña, A., y Navarro, A. (2014). Texto del estudiante. Ciencias de la Naturaleza 5o Primaria. Murcia: SM.

Quigley, C. F., y Herro, D. (2016). "Finding the Joy in the Unknown": Implementation of STEAM Teaching Practices in Middle School Science and Math Classrooms. Journal of Science Education and Technology, 25(3), 410-426. Retrieved from https://dx.doi.org/10.1007/s10956-016-9602-z 10.1007/s10956-016-9602-z Rossi-Cordero, A. E., y Barajas-Frutos, M. (2015). Gender imbalances in STEM career choice. Enseñanza de las Ciencias. Revista de investigación y experiencias didácticas, 33, 59-59. Retrieved from https://dx.doi.org/ 10.5565/rev/ensciencias. 1481 10.5565/rev/ensciencias. 1481

Sanders, M. (2009). Integrative stem education: Primer. The Technology Teacher, 68(4), 20-26.

Sauvé, L. (2000a). Para construir un patrimonio de investigación en Educación Ambiental. Tópicos en Educación Ambiental, 2(5), 51-69.

Sauvé, L. (2000b). Perspectivas curriculares para la formación de formadores en educación ambiental. Reflexiones sobre educación ambiental II: artículos publicados en la carpeta informativa del CENEAM, 219232.

Sheehan, K. J., Hightower, B., Lauricella, A. R., y Wartella, E. (2018). STEM Media in the Family Context: The Effect of STEM Career and Media Use on Preschoolers' Science and Math Skills. European Journal of STEMEducation, 3(3), 17-35. Retrieved from https://dx.doi.org/10.20897/ejsteme/3877 10.20897/ejsteme/3877 Son, J., y Diletti, J. (2017). What Can We Learn from Textbook Analysis? In E. J.-W. Son y T. W. y J.-J. Lo 
(Eds.), What Matters? Research Trends in International Comparative Studies in Mathematics Education (p.332). Springer.

Soria, A., Navarro, A., y López, S. (2014). Texto del estudiante. Ciencias de la Naturaleza 3o Primaria. Sevilla: SM.

STEM Integration in K-12 education: status, prospects, and an agenda for research. (2014). NRC.

Thibaut, L., Ceuppens, S., Loof, H. D., Meester, J. D., Goovaerts, L., Struyf, A., de Pauw, J. B., Dehaene, W., Deprez, J., Cock, M. D., Hellinckx, L., Knipprath, H., Langie, G., Struyven, K., de Velde, D. V., Petegem, P. V., y Depaepe, F. (2018). Integrated STEM Education: A Systematic Review of Instructional Practices in Secondary Education. European Journal of STEM Education, 3(1), 2-2. Retrieved from https://dx.doi.org/10.20897/ ejsteme/85525 10.20897/ejsteme/85525

Tristán-Mojica, M. A. (2013). Educación ambiental en la formación del docente de Primaria en la provincia de Veraguas-Panamá. Retrieved from https://dx.doi.org/10.14201/11586

Tsupros, N., Kohler, R., y Hallinen, J. (2008). STEM education: A project to identify the missing components. Intermediate Unit 1. In E. (Ed.), (p. 53-75). Pennilvania: Leonard Gelfand Center for Service Learning and Outreach.

Valdés, P., y Rojas, E. (2017). Texto del estudiante. Ciencias Naturales 5ํBásico. Santiago: Crecer Pensando. 